\title{
Correlated Equilibrium in Access Control for Wireless Communications
}

\author{
Eitan Altman ${ }^{1}$, Nicolas Bonneau ${ }^{1}$, and Mérouane Debbah ${ }^{2}$ \\ ${ }^{1}$ INRIA, Centre Sophia Antipolis, 2004 Route des Lucioles, B.P.93, \\ 06902 Sophia Antipolis, France \\ \{eitan.altman, nicolas.bonneau\}@sophia.inria.fr \\ ${ }^{2}$ Mobile Communications Group, Institut Eurecom, 2229 Route des Cretes, B.P.193, \\ 06904 Sophia Antipolis, France \\ merouane.debbah@eurecom.fr
}

\begin{abstract}
We study a finite population of mobiles communicating using the slotted ALOHA-type protocol. Our objective is the study of coordination between the mobiles in both cooperative as well as non-cooperative scenarios. Our study is based on the correlated equilibrium concept, a notion introduced by Aumann that broadens the Nash equilibrium. We study ways in which signaling can improve the performance both in the cooperative as well as in the non-cooperative cases, even in the absence of any extra information being conveyed through these signals.
\end{abstract}

\section{Introduction}

There has been a growing interest in studying competition (and also cooperation) aspects of networking in general, and of access to a common channel in particular. Non-cooperative game theory, as well as cooperative game theory, have been frequently used as a central framework for modeling such issues, see for example [1] and references therein.

We consider a finite population of mobile terminals that compete over the access to a common channel. The framework we consider is of a discrete time system (a simplified version of the slotted Aloha protocol [2]). All mobiles are thus supposed to be synchronized. As is frequently assumed when studying slotted Aloha, we assume that if more than one mobile attempts to send a packet at time slot $t$ then all transmitted packets are lost and mobiles wait a random amount of slots before retransmitting their packets, in order to avoid repeated collisions.

We consider both the cooperative as well as the non-cooperative approaches. For each case we study the impact of adding coordination mechanisms on the throughput.

The framework we consider is that of correlated games along with the notion of correlated equilibrium. The notion of correlated equilibrium was introduced by R. Aumann 1 in [3] and further studied in [4,5, 6. An algorithm for the

${ }^{1}$ Prof. R. Aumann has received in 2005 the Nobel prize in economy for his contributions to game theory, together with Thomas Schelling. 
computation of correlated equilibria is developed in [7]. Correlated equilibria are generalizations of the Nash equilibrium concept; the correlated equilibria are defined in a context where there is an arbitrator who can send (private or public) signals to the players. These signals allow players to coordinate their actions, and, in particular, to perform joint randomization over strategies.

In many contexts, an arbitrator is thought of as an intelligent entity, used for helping to solve conflicts and for proposing compromises to the different sides involved. In contrast, in correlated games, an arbitrator needs not have any intelligence. It is assumed to generate signals that do not depend on the system (or on individual) states. Moreover, it does not need to have any knowledge on the system. All the arbitrator has to do is to create some random signals (according to a randomized mechanism known by the players) that can help the synchronization (or coordination) between them.

In the context of non-cooperative games, each player has the possibility not to consider the signal(s) it receives. A multi-strategy obtained using the signals is a set of strategies (one strategy for each player which may depend on all the information available to the player including the signal it receives). It is said to be a correlated equilibrium (a precise definition will be given later) if no player has an incentive to deviate unilaterally from its part of the multi-strategy. A special type of "deviation" in this definition can be of course to ignore the signals.

An arbitrator may even be a virtual entity. As an example, the players can agree to use some random data (e.g., the first word they hear on the radio) as the signal or as an input to a function that allows to create a common signal (or a signal which may differ from one player to another).

Our contribution in this paper is not only in applying the notion of correlated equilibrium in the context of networking, but also in extending it to the multicriterion case; in our case, each mobile (player) has two objectives: expected throughput and expected power consumption. We use the correlated equilibrium setting adapted to the context of constrained optimization by each player (maximizing the average throughput with a constraint on the average power consumption).

Coordination between players turns out to be useful also in the case of cooperative optimization. Indeed, the coordination may be needed also in this framework in the so called team problem [1,8, i.e., where various players have the same common objective that they maximize (e.g., the global throughput). Users may benefit from performing joint randomizations, which may not be possible without coordination due to a possible distributed nature of the problem. The need for joint randomization in the team setting is due to the multi-objective nature of the problem (more precisely to the constraints on the expected power consumption).

The paper is organized as follows. The model is described in Sect. 2 The general game is analyzed in Sect. 3. We introduce a coordination mechanism, and we define and analyze the corresponding correlated strategies that arise in this context in Sect. 4. Finally, we present some results in Sect. 5] 


\section{The Model}

We consider a finite population of $m$ mobile terminals. Each mobile has a unique i.d. number ranging between 1 to $m$. Time is slotted.

Let $\mathcal{N}=\{0,1\}^{m}$ represent the set of all $2^{m}$ subsets of $\{1, \ldots, m\}$. At each time slot, a subset of mobiles $\mathbf{Z}(t) \in \mathcal{N}$ is assumed to be active. The number of active terminals at time $t$ is equal to the Hamming weight $|\mathbf{Z}(t)|=\sum_{i=1}^{m} Z_{i}(t)$ of $\mathbf{Z}(t)$ and denoted by $N(t)$. $\mathbf{Z}(t)$ (and thus $N(t)=|\mathbf{Z}(t)|$ ) are assumed to be stationary ergodic processes.

Each active mobile is assumed to be saturated, i.e., it always has packets to send. At each time slot, a random subset of mobiles is active. If at a time slot, more than one active mobile attempts to transmit then there is a collision and all packets transmitted in the time slot are lost.

Let $q_{i}$ denote the probability that mobile $i$ transmits a packet when active (we call $q_{i}$ the strategy). If $\mathbf{z} \in \mathcal{N}$, let $\zeta(\mathbf{z})$ be the probability that the subset $\mathbf{z}$ of mobiles is active at a slot and let $\pi_{n}=\sum_{|\mathbf{z}|=n} \zeta(\mathbf{z})$ be the probability that there are $n$ active mobiles at a slot. In particular, the probability that mobile $i$ is the only active mobile in a slot is $\zeta\left(\mathbf{e}_{i}\right)$ where $\mathbf{e}_{i}$ is the vector whose elements are all zero except for the $i$ th entry which equals one.

The probability of a successful transmission at a time slot is

$$
\begin{aligned}
\Theta_{\text {all }}\left(q_{1}, \ldots, q_{m}\right) & =\mathbb{E}_{\mathbf{Z}}\left[\sum_{i \in \mathbf{Z}} q_{i} \prod_{j \in \mathbf{Z} \backslash\{i\}}\left(1-q_{j}\right)\right] \\
& =\sum_{\mathbf{z} \in \mathcal{N}} \zeta(\mathbf{z}) \sum_{i \in \mathbf{z}} q_{i} \prod_{j \in \mathbf{z} \backslash\{i\}}\left(1-q_{j}\right) .
\end{aligned}
$$

which is also the system throughput. The expected average throughput per mobile is $\Theta_{\text {all }} / m$. The throughput of mobile $i$ conditioned on being active is given by

$$
\begin{aligned}
\Theta_{i}^{\text {act }}\left(q_{1}, \ldots, q_{m}\right) & =\mathbb{E}_{\mathbf{Z}}\left[q_{i} \prod_{j \in \mathbf{Z} \backslash\{i\}}\left(1-q_{j}\right) \mid i \in \mathbf{Z}\right] \\
& =q_{i} \sum_{\substack{\mathbf{z} \in \mathcal{N} \\
i \in \mathbf{Z}}} \zeta(\mathbf{z}) \prod_{j \in \mathbf{Z} \backslash\{i\}}\left(1-q_{j}\right) .
\end{aligned}
$$

In the following, the purpose of cooperative optimization will be to maximize the system throughput $\Theta_{\text {all }}$, whereas in a non-cooperative setting, each mobile will attempt to maximize selfishly its conditional throughput $\Theta_{i}^{\text {act }}$, which we call its utility.

\section{No Coordination Mechanism}

\subsection{General Case}

The maximal throughput that can be attained is obtained by maximizing the system throughtput $\Theta_{\text {all }}\left(q_{1}, \ldots, q_{m}\right)$ given by (1) over $\left(q_{1}, \ldots, q_{m}\right) \in[0,1]^{m}$. Since 
$\Theta_{\text {all }}\left(q_{1}, \ldots, q_{m}\right)$ is a multivariate polynomial, hence continuous in $\left(q_{1}, \ldots, q_{m}\right)$, and $[0,1]^{m}$ is a compact set, the existence of a maximum is immediate. For given $\{\zeta(\mathbf{z}) / \mathbf{z} \in \mathcal{N}\}$, computing this maximum is a constrained optimization problem [9].

If the mobiles are non-cooperative and care only for their own throughput then it is immediate from (2) that the only Nash equilibrium 2 is where all mobiles transmit with $q_{i}=1$. The global throughput is then $\pi_{1}$ and the expected average throughput per mobile is $\pi_{1} / \mathrm{m}$.

In the non-cooperative case, we are also interested by the conditional throughput, i.e., the throughput of a mobile averaged over the activity periods of the mobile. The conditional throughput of mobile $i$ when $q_{i}=1$ for all mobiles is given by $\zeta\left(\mathbf{e}_{i}\right)$.

\subsection{Power Considerations}

In reality mobile users are sensitive to power consumption. Their objective is to maximize the system throughput (in the cooperative case) or the individual throughput (in the non-cooperative case) under the constraints $q_{i} \leq q_{i}^{\max }$ for some constant $q_{i}^{\max }$, for all users $i$. In the cooperative case, we can model the choice of transmission probability $q_{i}$ as a constrained optimization problem. In the non-cooperative case, it is easy to see that the Nash equilibrium is obtained with $q_{i}=q_{i}^{\max }$ for all mobiles. From (1), this gives at the Nash equilibrium the throughput of

$$
\Theta_{\mathrm{all}}\left(q_{1}^{\max }, \ldots, q_{m}^{\max }\right)=\sum_{\mathbf{z} \in \mathcal{N}} \zeta(\mathbf{z}) \sum_{i \in \mathbf{z}} q_{i}^{\max } \prod_{j \in \mathbf{z} \backslash\{i\}}\left(1-q_{j}^{\max }\right),
$$

and from (2), the conditional throughput as

$$
\Theta_{i}^{\mathrm{act}}\left(q_{1}^{\max }, \ldots, q_{m}^{\max }\right)=q_{i}^{\max } \sum_{\substack{\mathbf{z} \in \mathcal{N} \\ i \in \mathbf{z}}} \zeta(\mathbf{z}) \prod_{j \in \mathbf{z} \backslash\{i\}}\left(1-q_{j}^{\max }\right) .
$$

\section{Coordination, Correlated Equilibrium and Optimization}

\subsection{Coordination Mechanism}

If the base station had full information and could schedule transmissions of the mobiles then full utilisation (i.e., a throughput of $1-\pi_{0}$ ) could be achieved by a TDMA type approach. We consider however the case where the base station has no control over the mobiles and has no information on their power constraints nor on their number. It can only serve as an arbitrator, in the sense that was discussed in the introduction.

${ }^{2}$ A Nash equilibrium is a set of strategies such that no mobile can improve its utility by deviating unilaterally from its strategy. 
We therefore consider the following coordination mechanism. We assume that at each time slot $t$, the base station can send a signal to all mobiles in the form of a random variable $X(t)$, uniformly distributed over the integers $\{0, \ldots, K-1\}$ for some integer $K \geq 2$. We assume for simplicity that $m$ is a multiple of $K$. The process $X(t)$ is assumed to be independent of $\mathbf{Z}(t)$.

\subsection{Transmission Strategy for Mobiles}

In absence of any coordination mechanism, a strategy of a mobile would be the probability of transmitting a packet. In the presence of the coordination mechanism, a mobile has the possibility to use a larger notion of strategies.

Definition 1. We define the set of correlated policies as follows.

- We partition the set of all mobiles into $K$ subgroups $S_{j}, j=1, \ldots, K$ where $S_{j}$ contains a mobile $i$ if and only if $i=j-1(\bmod K)$ (denoted $\left.i \equiv j-1\right)$.

- A correlated strategy of a mobile is described using two real numbers in the unit interval: $p_{i}$ and $q_{i}$.

- At time $t$, an active mobile $i$ transmits a packet with probability $p_{i}$ if and only if $i \in S_{X(t)}$. Otherwise it transmits with probability $q_{i}$.

Note that this class of correlated strategies includes in particular the noncorrelated strategies. Thus, in the non-cooperative setting, a mobile has always the possibility of ignoring the signals $X(t)$ by using $p_{i}=q_{i}$. The latter can be viewed as a non-correlated strategy.

We call $\left(p_{i}, q_{i}\right)$ the strategy of mobile $i$. For two $m$-dimensional vectors $\mathbf{p}$ and $\mathbf{q}$ we define $(\mathbf{p}, \mathbf{q})$ to be a multi-strategy for all mobiles, where mobile $i$ uses the $i$ th entry $\left(p_{i}, q_{i}\right)$ of the vectors $(\mathbf{p}, \mathbf{q})$. Let

$$
\mathcal{U}=\left\{(\mathbf{p}, \mathbf{q}) / \forall i \in\{1, \ldots, m\}, p_{i} \in[0,1], q_{i} \in[0,1]\right\}
$$

denote the class of all multi-strategies.

Define $(\mathbf{p}, \mathbf{q})^{-i}$ to be the set of $m-1$ strategies of all mobiles except for mobile $i$, and set $\left((\mathbf{p}, \mathbf{q})^{-i},\left(p^{\prime}, q^{\prime}\right)_{i}\right)$ to be the policy where all mobiles other than the $i$ th one use the policies described by $(\mathbf{p}, \mathbf{q})^{-i}$ whereas the $i$ th mobile uses policy $\left(p^{\prime}, q^{\prime}\right)$.

\subsection{Power Considerations}

We assume that mobile $i$ has a constraint on the average power it can use while active. More precisely, the average power consumption during activity periods of a mobile with parameters $(p, q)$ is

$$
\operatorname{Pow}(p, q)=\frac{p}{K}+\frac{(K-1) q}{K} .
$$

We then assume that mobile $i$ has the power constraint

$$
\operatorname{Pow}\left(p_{i}, q_{i}\right) \leq q_{i}^{\max } \text { where } q_{i}^{\max } \leq 1 .
$$


Let $U_{i}^{\text {cons }}$ denote the class of strategies of mobile $i$ satisfying (4). Let

$$
\mathcal{U}^{\text {cons }}=\left\{\mathbf{u} \in \mathcal{U} / \forall i \in\{1, \ldots, m\}, u_{i} \in U_{i}^{\text {cons }}\right\}
$$

denote the class of multi-strategies $\mathbf{u}$ for which for each $i, u_{i}=\left(p_{i}, q_{i}\right)$ satisfies (41).

Definition 2. A multi-strategy $\mathbf{u} \in \mathcal{U}^{\text {cons }}$ is said to be a correlated equilibrium if for all $i$ and $\left(p^{\prime}, q^{\prime}\right) \in U_{i}^{\text {cons }}$

$$
\Theta_{i}^{a c t}(\mathbf{u}) \geq \Theta_{i}^{a c t}\left(\mathbf{u}^{-i},\left(p^{\prime}, q^{\prime}\right)_{i}\right)
$$

Definition 3. A multi-strategy $\mathbf{u}^{*} \in \mathcal{U}^{\text {cons }}$ is said to be correlated optimal if for all feasible multi-strategies $\mathbf{u} \in \mathcal{U}^{\text {cons }}$,

$$
\Theta_{\text {all }}\left(\mathbf{u}^{*}\right) \geq \Theta_{\text {all }}(\mathbf{u}) .
$$

The expressions for $\Theta_{\text {all }}(\mathbf{u})$ and $\Theta_{i}^{\text {act }}(\mathbf{u})$ can be written as

$$
\begin{aligned}
\Theta_{\text {all }}(\mathbf{u})= & \sum_{\mathbf{z} \in \mathcal{N}} \zeta(\mathbf{z}) \sum_{i \in \mathbf{z}}\left(\frac{p_{i}}{K} \prod_{\substack{j \in \mathbf{z} \backslash\{i\} \\
j \equiv i}}\left(1-p_{j}\right) \prod_{\substack{j \in \mathbf{z} \backslash\{i\} \\
j \neq i}}\left(1-q_{j}\right)\right. \\
& \left.+\frac{q_{i}}{K} \sum_{\substack{k=1 \\
k \neq i}}^{K} \prod_{\substack{j \in \mathbf{z} \backslash\{i\} \\
j \equiv k}}\left(1-p_{j}\right) \prod_{\substack{j \in \mathbf{z} \backslash\{i\} \\
j \neq k}}\left(1-q_{j}\right)\right)
\end{aligned}
$$

and

$$
\begin{aligned}
\Theta_{i}^{\mathrm{act}}(\mathbf{u})= & \frac{p_{i}}{K} \sum_{\substack{\mathbf{z} \in \mathcal{N} \\
i \in \mathbf{z}}} \zeta(\mathbf{z}) \prod_{\substack{j \in \mathbf{z} \backslash\{i\} \\
j \equiv i}}\left(1-p_{j}\right) \prod_{\substack{j \in \mathbf{z} \backslash\{i\} \\
j \neq i}}\left(1-q_{j}\right) \\
& +\frac{q_{i}}{K} \sum_{\substack{\mathbf{z} \in \mathcal{N} \\
i \in \mathbf{z}}} \zeta(\mathbf{z}) \sum_{\substack{k=1 \\
k \neq i}} \prod_{\substack{j \in \mathbf{z} \backslash\{i\} \\
j \equiv k}}\left(1-p_{j}\right) \prod_{\substack{j \in \mathbf{z} \backslash\{i\} \\
j \neq k}}\left(1-q_{j}\right) .
\end{aligned}
$$

$\Theta_{i}^{\text {act }}(\mathbf{u})$ is an affine function of $p_{i}$ and $q_{i}$. Therefore, in order to maximize $\Theta_{i}^{\text {act }}(\mathbf{u})$, the inequality in (4) will be an equality: each mobile will transmit at the maximum of its possibilities. In the next section, we investigate how the power is split between $p_{i}$ and $q_{i}$ for each mobile in a particular case.

\subsection{Symmetric Case}

Solving the constrained optimization problems of (11) or (7), as well as finding Nash or correlated equilibria, becomes rapidly intractable in the general case when the number of mobiles $m$ (and hence the number of variables in the multivariate polynomials involved) increases. To simplify the analysis, we consider 
a symmetric case when the coefficients $\zeta(\mathbf{z})$ depend only on $|\mathbf{z}|$, and the power constraints $q_{i}^{\max }=q^{\max }$ are the same for all users.

We consider a simple model when mobiles are independently active with a probability $\pi$. This corresponds to the model used in [10] for users with a single packet buffer, when the probability of arrival of a new packet is equal to the probability of retransmission of a backlogged packet. In this case, the coefficients in (11) and (7) become symmetric, since for all $\mathbf{z}$ such that $|\mathbf{z}|=n, \zeta(\mathbf{z})$ are equal:

$$
\zeta(\mathbf{z})=\pi^{|\mathbf{z}|}(1-\pi)^{m-|\mathbf{z}|}
$$

In the non-cooperative case, we can restrict to the same strategy $(p, q)$ being used by all users, and investigate if a single user deviating from this strategy benefits by using a different strategy $(\hat{p}, \hat{q})$. Recall that $\pi_{n}=\sum_{|\mathbf{z}|=n} \zeta(\mathbf{z})$. Let

$$
\ell=\frac{m}{K}, \quad \lambda=m-\frac{m}{K} .
$$

After some manipulations, (8) can be rewritten as:

$$
\begin{aligned}
\Theta^{\mathrm{act}}(\mathbf{u}) & =\frac{\hat{p}}{K} \sum_{n=1}^{m} \frac{1}{\left(\begin{array}{c}
m \\
n
\end{array}\right)} \pi_{n} \\
& \times \sum_{k=\max (0, n-1-\lambda)}^{\min (\ell-1, n-1)}\left(\begin{array}{c}
\ell-1 \\
k
\end{array}\right)\left(\begin{array}{c}
\lambda \\
n-1-k
\end{array}\right)(1-p)^{k}(1-q)^{n-1-k} \\
& +\frac{(K-1) \hat{q}}{K} \sum_{n=1}^{m} \frac{1}{\left(\begin{array}{c}
m \\
n
\end{array}\right)} \pi_{n} \\
& \times \sum_{k=\max (0, n-\lambda)}^{\min (\ell, n-1)}\left(\begin{array}{l}
\ell \\
k
\end{array}\right)\left(\begin{array}{c}
\lambda-1 \\
n-1-k
\end{array}\right)(1-p)^{k}(1-q)^{n-1-k} .
\end{aligned}
$$

The power constraints (4) give us

$$
\hat{p}=K q^{\max }-(K-1) \hat{q} .
$$

Replacing $\hat{p}$ by this expression in (10), we obtain $\Theta^{\text {act }}(\mathbf{u})$ as an affine function in $\hat{q}$. Hence, the optimal $\hat{q}$ will be either

$$
\max \left(0, \frac{K q^{\max }-1}{K-1}\right) \text { or } \min \left(1, \frac{K q^{\max }}{K-1}\right)
$$

depending on the sign of the coefficient

$$
\begin{aligned}
& \sum_{n=1}^{m} \frac{1}{\left(\begin{array}{c}
m \\
n
\end{array}\right)} \pi_{n} \sum_{k=\max (0, n-\lambda)}^{\min (\ell, n-1)}\left(\begin{array}{l}
\ell \\
k
\end{array}\right)\left(\begin{array}{c}
\lambda-1 \\
n-1-k
\end{array}\right)(1-p)^{k}(1-q)^{n-1-k} \\
& -\sum_{n=1}^{m} \frac{1}{\left(\begin{array}{c}
m \\
n
\end{array}\right)} \pi_{n} \sum_{k=\max (0, n-1-\lambda)}^{\min (\ell-1, n-1)}\left(\begin{array}{c}
\ell-1 \\
k
\end{array}\right)\left(\begin{array}{c}
\lambda \\
n-1-k
\end{array}\right)(1-p)^{k}(1-q)^{n-1-k} .
\end{aligned}
$$


This gives us a simple formula to investigate whether or not a given value of $(p, q)$ that saturates (44) is a correlated equilibrium: replace $(p, q)$ by their values in (12) and estimate the sign of the expression. If the chosen $q$ satisfies

$$
q=\max \left(0, \frac{K q^{\max }-1}{K-1}\right)
$$

and the sign of (12) is negative or if the chosen $q$ satisfies

$$
q=\min \left(1, \frac{K q^{\max }}{K-1}\right)
$$

and the sign of (12) is positive, then $(p, q)$ is indeed a correlated equilibrium.

\section{$5 \quad$ Results}

We use the terms cooperative and non-cooperative to describe the behavior of mobiles, whereas the term coordination refers to the presence of a common signal. Without coordination, the equilibrium concept in the non-cooperative case is the Nash equilibrium, whereas it is the correlated equilibrium with coordination.

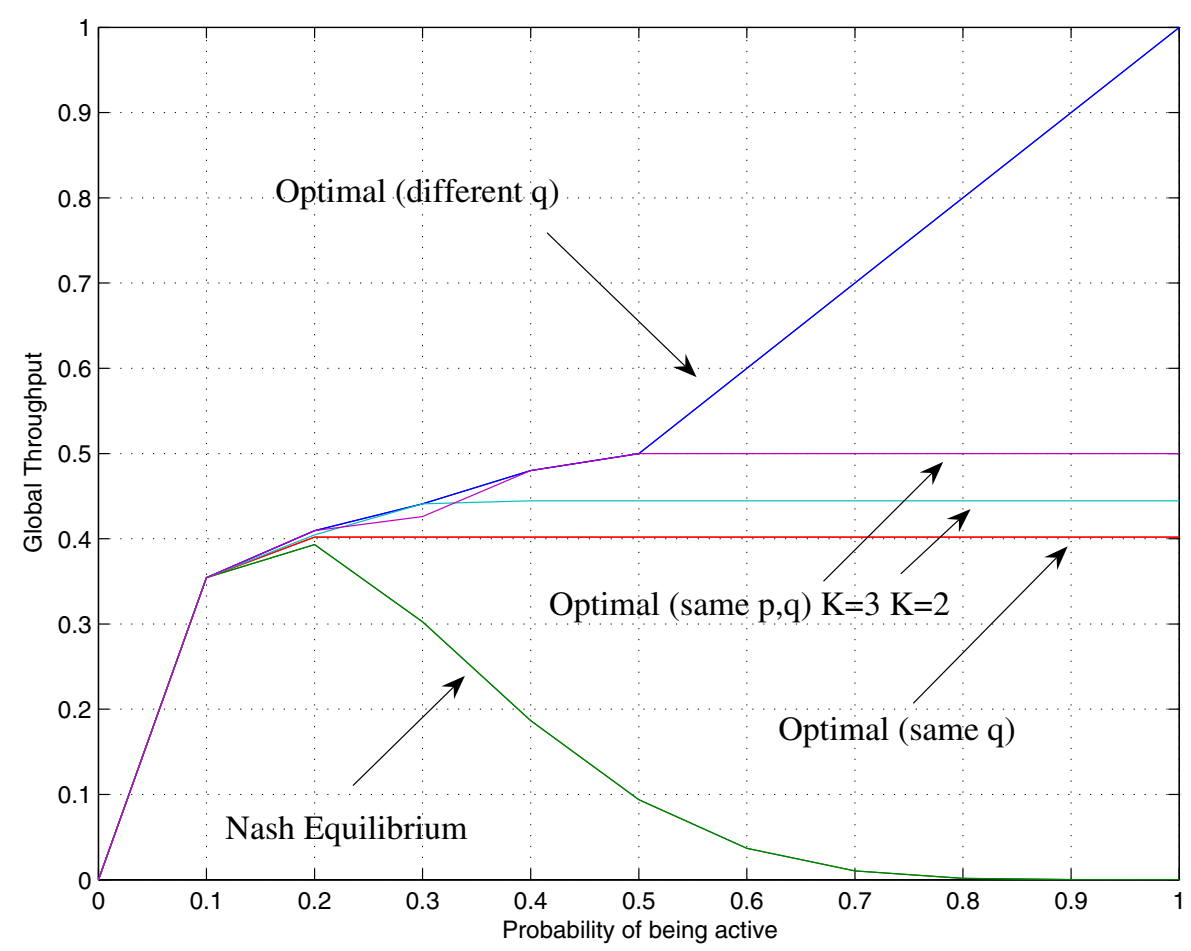

Fig. 1. System throughput versus the probability of being active for a mobile with and without coordination, for 6 users, without power constraints 


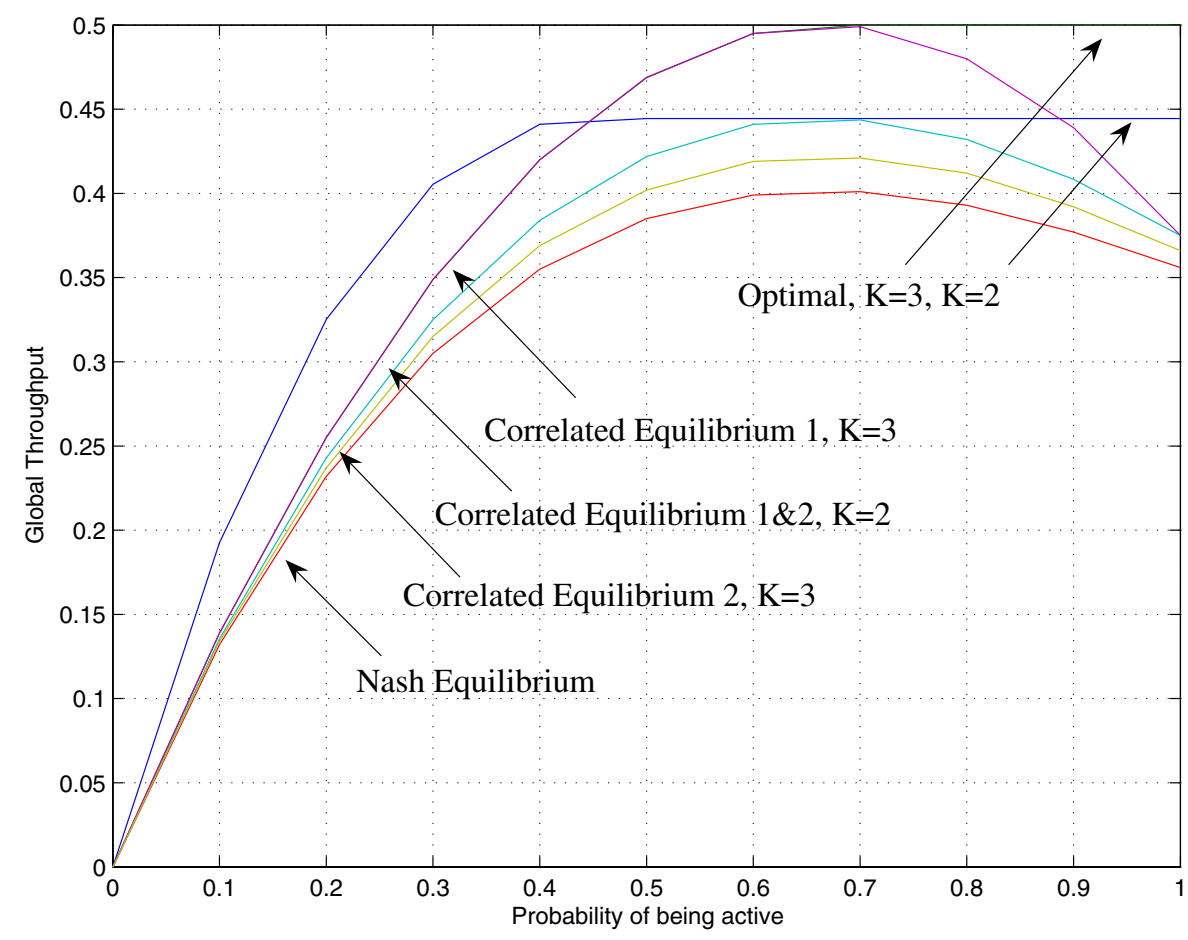

Fig. 2. System throughput versus the probability of being active for a mobile with power constraint $q^{\max }=0.25$, for 6 users

In this section, we consider the setting of Subsect.4.4. However, an interesting result is that, even in this symmetric case, the optimal throughput is neither reached by saturating the power constraints $q_{i}^{\max }$ for all users nor for a symmetric attribution of the channel (i.e., the same strategy for all users).

In Fig. 1, we have plotted the system throughput $\Theta_{\text {all }}$ versus the probability of being active $\pi$ with and without coordination, according to (1) and (7), for 6 users, without power constraints $\left(q_{i}^{\max }=1\right.$ for all users $)$. We observe that the optimal throughput with the same strategy for all mobiles reaches a plateau and stays constant, no matter how active the mobiles are. With coordination, the value of this plateau is increased.

With a non-symmetric attribution of the strategies, a higher system throughput can be achieved. The linear portion of the curve, for $\pi>0.5$, is actually obtained by letting only one user transmit; for $\pi \leq 0.5$, it is optimal to let several users transmit. Without power constraints, the optimal throughput with coordination is the same as without coordination.

The system throughput reached at Nash equilibrium (i.e., $q=1$ for all mobiles) is close to the optimum for low values of $\pi$ (when few mobiles are active), but rapidly decreases and approaches 0 as the probability of being active increases. Note that without power constraints, Nash and correlated equilibrium 
coincide, therefore the coordination mechanism does not increase the throughput in the non-cooperative case. We remark that the curve for Nash equilibrium corresponds to the throughput calculated in [10], which is simply $m \pi(1-\pi)^{m-1}$.

The optimal curves in Fig. 1 are obtained without power constraints, therefore with power constraints the optimal curves will always be lower.

In Fig. 2, we have plotted the system throughput $\Theta_{\text {all }}$ obtained in the correlated equilibrium with power constraint $q^{\max }=0.25$. In the case $K=3$, two correlated equilibria are possible: $p=0.75, q=0$ or $p=0, q=0.375$ (denoted respectively as 1 and 2 in the figure). In the case $K=2$, there are two correlated equilibria as well: $p=0.5, q=0$ and $p=0, q=0.5$ (both give the same system throughput). As a comparison, we have plotted the optimal throughput that can be obtained under the power constraint $q^{\max }=0.25$ in the cooperative case, as well as the throughput obtained in the Nash equilibrium without coordination.

Non-cooperative throughput is improved compared to the case without power constraints. With strong power constraints, we observe that the coordination mechanism allows to obtain higher values of the throughput in the non-cooperative case. For some probabilities $\pi$, non-cooperative global throughput almost reaches the values obtained in the cooperative case.

\section{Conclusion}

We have investigated a game theoretical setting including a coordination mechanism in a distributed access control. Our analysis is based on the concept of correlated equilibrium that enriches the strategies of mobiles. Power constraints are primordial in order to give a sense to coordination. In the absence of power constraints, coordination does not necessarily improve the channel utilization. The proposed coordination mechanism can improve the utilization of the channel in presence of power constraints, even in presence of selfish users.

\section{References}

1. Altman, E., Boulogne, T., Azouzi, R.E., Jimenez, T., Wynter, L.: A survey on networking games. Computers and Operations Research (2005)

2. Roberts, L.: Aloha Packet System with and without Slots and Capture. Technical report, Stanford Research Institute, Advanced Research Projects Agency, Network Information Center (1972)

3. Aumann, R.: Subjectivity and Correlation in Randomized Strategies. Journal of Mathematical Economics 1 (1974) 67-96

4. Aumann, R.: Correlated Equilibrium as an Expression of Bayesian Rationality. Journal of Mathematical Economics 55 (1987) 1-18

5. Hart, S., Schmeidler, D.: Existence of Correlated Equilibria. Mathematics of Operations Research 14(1) (1989) 18-25

6. Neyman, A.: Correlated Equilibrium and Potential Games. International Journal of Game Theory 26 (1997) 223-227

7. Papadimitriou, C.H.: Computing Correlated Equilibria in Multiplayer Games. (Available on the author's home page: http://www.cs.berkeley.edu/ christos) 
8. Başar, T., Cruz, J.B. In: Concepts and methods in multiperson coordination and control. North-Holland Publishing Company (1982) 351-394

9. Luenberger, D.: Linear and Nonlinear Programming. 2nd edn. Addison-Wesley, Inc., Reading, Massachusetts (1984)

10. Kleinrock, L., Lam, S.: Packet Switching in a Multiaccess Broadcast Channel: Performance Evaluation. IEEE Trans. on Communications (4) (1975) 410-423 